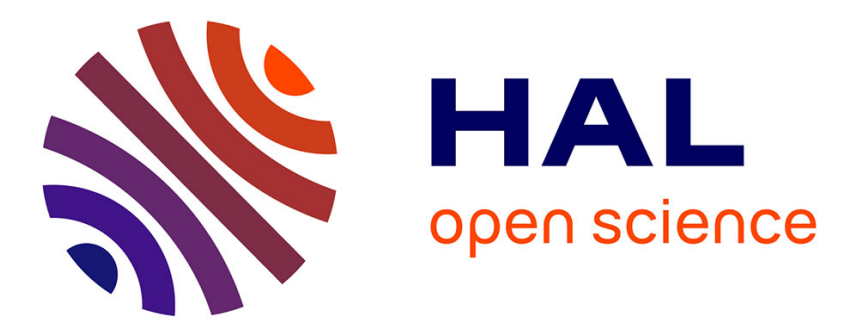

\title{
Transient growth of perturbations in Stokes oscillatory flows
}

\author{
Damien Biau
}

\section{To cite this version:}

Damien Biau. Transient growth of perturbations in Stokes oscillatory flows. Journal of Fluid Mechanics, 2016, 794, pp.10. 10.1017/jfm.2016.210 . hal-01377582

\section{HAL Id: hal-01377582 \\ https://hal.science/hal-01377582}

Submitted on 7 Oct 2016

HAL is a multi-disciplinary open access archive for the deposit and dissemination of scientific research documents, whether they are published or not. The documents may come from teaching and research institutions in France or abroad, or from public or private research centers.
L'archive ouverte pluridisciplinaire HAL, est destinée au dépôt et à la diffusion de documents scientifiques de niveau recherche, publiés ou non, émanant des établissements d'enseignement et de recherche français ou étrangers, des laboratoires publics ou privés. 


\title{
Transient growth of perturbations in Stokes oscillatory flows
}

\author{
Damien Biau† \\ DynFluid Laboratory, Arts et Métiers ParisTech, 151 Boulevard de l'Hôpital, 75013 Paris, France
}

Oscillatory Stokes flows, with zero mean, are subjected to subcritical transition to turbulence. The maximal energy growth of perturbations is computed in the subcritical regime through an optimisation method. The results show strong amplifications during half a period. The energy transfer from the base flow involves an Orr mechanism with two-dimensional vorticity waves, and the maximum energy scales exponentially with the Reynolds number. Nonlinear simulations show that low-energy perturbations are sufficient to trigger turbulent flow.

Key words: boundary layer stability, instability, transition to turbulence

\section{Introduction}

In the framework of linear stability analysis, time-periodic flows can be classified into oscillatory flows (with zero mean) and pulsatile flows (with non-zero mean). In the latter case, the stability analysis of frozen base flow leads to accurate results, provided that the instability grows on a time scale much smaller than the period of the base flow. Therefore, the modulation of the mean flow can be considered as a secondary effect since the oscillatory part is small compared with the mean. However, for strict oscillatory cases, the time variation of the base flow is a fundamental effect and the quasisteady approximation becomes irrelevant.

The Stokes boundary layer is one of the simplest oscillatory shear flows. A sinusoidal pressure gradient, or a sinusoidal wall motion, is imposed on a fluid at rest in a semi-infinite domain. The three relevant physical parameters are the kinematic viscosity $v$, the period of oscillations $T$ and the amplitude of velocity variations $U_{0}$. The control parameter, namely the Reynolds number, takes the form

$$
\operatorname{Re}=U_{0} \sqrt{T / \nu}
$$

which represents the ratio of transient inertial force and viscous shear force.

$†$ Email address for correspondence: damien.biau@ensam.eu 


\section{Biau}

Von Kerczek \& Davis (1974) and Hall (1978) performed the first modal analysis, based on Floquet theory, but due to limited computer capability it was not possible to reach high enough Reynolds number values to find an instability. Some decades later Blennerhassett \& Bassom (2002) found the critical Reynolds number, $\operatorname{Re}_{c}=2511$. However, that critical value is well above the thresholds observed in experiments; these discrepancies are thoroughly reviewed and discussed in Blennerhassett \& Bassom (2008). Ozdemir, Hsu \& Balachandar (2014) have performed direct numerical simulations, and they confirm the observation of turbulent flows for Reynolds numbers above $\operatorname{Re}_{T}=900$. A subcritical transition to turbulence is typical for wall-bounded shear flows, since this property is related to the non-normal nature of the linearised Navier-Stokes equations (Farrell 1988; Butler \& Farrell 1992; Trefethen et al. 1993; Schmid \& Henningson 1999; Schmid 2007). Consequently, the idealised analytical flow has quite different stability properties from those seen in practice. For example, the perturbation growth can be amplified by wall roughness (Blondeaux \& Vittori 1994; Vittori \& Verzicco 1998) or a base flow variation (Thomas et al. 2015).

While the Floquet analysis (Blennerhassett \& Bassom 2002) focuses on the asymptotic behaviour determined by the least stable mode, a general small disturbance is in fact a weighted combination of all eigenmodes. Moreover, because of the non-normality of the linearised stability operator, there is the potential for very large transient amplification of the disturbance energy, even in nominally stable flow conditions (Akhavan, Kamm \& Shapiro 1991). The kinematic mechanism for the initial amplification of disturbance travelling with the shear flow was initially discovered by Orr (1907). In order to understand how an asymptotically stable flow becomes turbulent, Orr considered the stability analysis as an initial value problem. The result is a possibility of linear transient growth, eventually followed by an asymptotic (modal) decay. It is inferred that an initial growth could lead to disturbances so large as to trigger a nonlinear self-sustaining process, thereby triggering the turbulence. Nonetheless, the short-time dynamics raises the difficulty of determining the initial condition. As a solution, Farrell (1988) computed the optimal perturbation, namely the initial condition that maximises the energy gain.

The present paper aims to apply this strategy to the subcritical transition in Stokes boundary layer flows. The optimisation method was developed by Andersson, Berggren \& Henningson (1999), Corbett \& Bottaro (2000) and Luchini (2000), and is briefly recalled in $\S 2$ for consistency; the results are presented in $\S 3$. A critical discussion and some possible extensions are proposed in the conclusion.

\section{Method}

The Navier-Stokes equations are made dimensionless with the following characteristic scales: the period of oscillations $T$, the amplitude of the velocity $U_{0}$ and the diffusive length scale $\delta=\sqrt{v T}$. The flow is described by Cartesian coordinates $(x, y, z)$, respectively the streamwise, wall-normal and spanwise directions. The configuration allows for two analytical solutions (Schlichting 1979).

In the case of an oscillating wall, the solution (obtained by Stokes) is

$$
U(y, t)=\cos (2 \pi t-\sqrt{\pi} y) \mathrm{e}^{-\sqrt{\pi} y} .
$$

In the case of an oscillating pressure gradient, the solution is

$$
U(y, t)=\cos (2 \pi t)-\cos (2 \pi t-\sqrt{\pi} y) \mathrm{e}^{-\sqrt{\pi} y} .
$$

These two solutions ((2.1) and (2.2)) are shown in figure 1. 
(a)

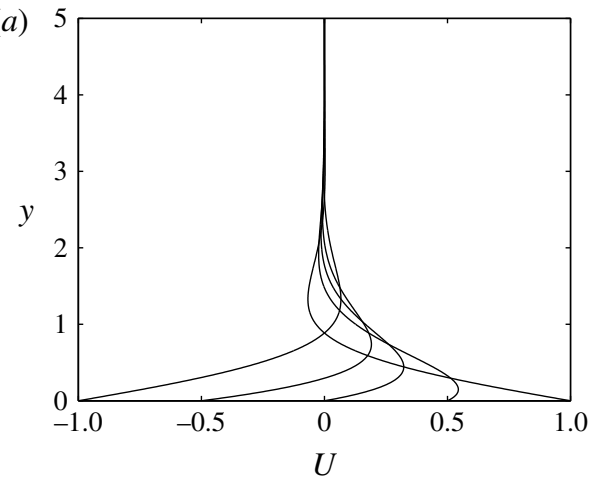

(b)

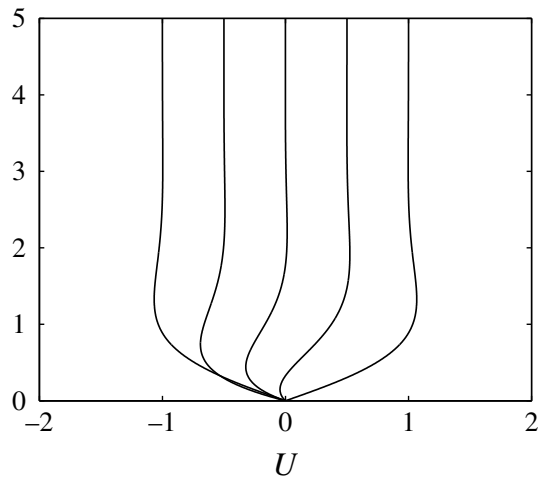

FIGURE 1. Laminar solution during half a period, for an oscillating wall $(a)$ and oscillating external flow $(b)$.

The Navier-Stokes equations, linearised around the laminar state $U(y, t)$, are

$$
\left.\begin{array}{c}
\nabla \cdot \boldsymbol{u}=0 \\
\boldsymbol{u}_{t}+\operatorname{Re}(\boldsymbol{U} \cdot \nabla \boldsymbol{u}+\boldsymbol{u} \cdot \nabla \boldsymbol{U})=-\nabla p+\nabla^{2} \boldsymbol{u} .
\end{array}\right\}
$$

The Reynolds number is defined by (1.1). Perturbations $(\boldsymbol{u}=[u, v, w])$ are decomposed into the sum of Fourier modes in homogeneous directions, and the linear framework allows mode-by-mode analysis, $\boldsymbol{u}(y, t) \mathrm{e}^{\mathrm{i}\left(k_{x} x+k_{z} z\right)}, k_{x}$ and $k_{z}$ being respectively the streamwise and spanwise wavenumbers. Equations (2.3) are associated with no-slip boundary conditions at the wall $(y=0)$,

$$
\boldsymbol{u}=0 \text {, }
$$

and an irrotational flow condition in the far field $(y \rightarrow \infty)$,

$$
\frac{\partial \boldsymbol{u}}{\partial y}=-\sqrt{k_{x}^{2}+k_{z}^{2}} \boldsymbol{u} .
$$

The non-dimensional kinetic energy is such that

$$
E(t)=\int_{y} \frac{\boldsymbol{u}^{*} \cdot \boldsymbol{u}}{2} \mathrm{~d} y,
$$

with * denoting complex conjugate.

The energy equation for the perturbations is obtained from the integrated momentum equation,

$$
\frac{\mathrm{d} E}{\mathrm{~d} t}=-\operatorname{Re} \int_{y=0}^{\infty} \overline{u v} \frac{\partial U}{\partial y} \mathrm{~d} y-\int_{y=0}^{\infty} u_{i, j} u_{i, j} \mathrm{~d} y .
$$

The first right-hand-side term is the production term, where $\overline{u v}$ represents the average along the homogeneous directions $(x, z)$ of the product of the streamwise and wall-normal velocities, and $\partial U / \partial y$ denotes the laminar shear. The last term, always negative, designates the dissipation of kinetic energy by the viscous effect.

From (2.7), it can be stated that the two Stokes solutions ((2.1) and (2.2)) lead to identical energy growth. In particular, this is true for the less stable mode, as shown, with a different argument, by Blennerhassett \& Bassom (2002): they proved that eigenvalues are invariant under the transformation $U(y, t) \rightarrow U(y, t)-\cos (2 \pi t)$. 


\section{Biau}

Given that the basic state can grow (or decay) simultaneously with the growth of a disturbance, it is sometimes more appropriate to consider a relative criterion for instability defined as the energy ratio of the perturbation to the base flow (Conrad \& Criminale 1965). This is especially true for the case where the kinetic energy of the base flow would become null, so that the infinitesimal assumption for the perturbation would become questionable. However, this consideration is not significant in the present case because the energy of the laminar state is strictly positive.

The concept of optimal perturbations was first introduced by Farrell (1988) and further developed by Butler \& Farrell (1992). The method was initially based on the computation of the full spectrum of the linearised Navier-Stokes operator. A significant improvement was proposed by Andersson et al. (1999), Corbett \& Bottaro (2000) and Luchini (2000), with an optimisation based on the continuous adjoint operator. This method, initially used for steady base flow, can easily be applied to base flows with arbitrary time dependence (Zhao, Ghidaoui \& Kolyshkin 2007). The applications of adjoint methods for linear stability analysis are reviewed in depth by Luchini \& Bottaro (2014).

If we formally denote by $L \boldsymbol{u}=0$ the linearised equations (2.3), then the optimisation problem is to maximise the energy $E(t)$ subject to the constraint $L \boldsymbol{u}=0$. The Lagrangian functional is based on an energy-like norm and reads

$$
\mathscr{L}\left(\boldsymbol{u}, \boldsymbol{u}^{\dagger}\right)=E(t)+\int_{V} \boldsymbol{u}^{\dagger *} \cdot(L \boldsymbol{u}) \mathrm{d} V
$$

with $\boldsymbol{u}^{\dagger}$ the Lagrange multiplier. The optimal state is reached for the stationary point for the Lagrange function, which gives us the adjoint problem:

$$
\left.\begin{array}{c}
\nabla \cdot \boldsymbol{u}^{\dagger}=0, \\
-\boldsymbol{u}_{t}^{\dagger}-\operatorname{Re}\left(\boldsymbol{U} \cdot \nabla \boldsymbol{u}^{\dagger}-\boldsymbol{u}^{\dagger} \cdot(\nabla \boldsymbol{U})^{\mathrm{T}}\right)=-\nabla p^{\dagger}+\nabla^{2} \boldsymbol{u}^{\dagger} .
\end{array}\right\}
$$

The adjoint equations (2.9) are associated with the following boundary conditions: noslip conditions at the wall,

$$
\boldsymbol{u}^{\dagger}=0
$$

and an irrotational flow condition in the far field,

$$
\frac{\partial \boldsymbol{u}^{\dagger}}{\partial y}=-\sqrt{k_{x}^{2}+k_{z}^{2}} \boldsymbol{u}^{\dagger}
$$

Practically, to identify the flow state at $t=T_{0}$ producing the largest disturbance growth at any given $T_{f}>T_{0}$, a repeated numerical integration of the direct and adjoint equations is used, coupled with transfer conditions. The direct problem (the linearised Navier-Stokes equations) is integrated from a given initial condition at $t=T_{0}$ up to the final target time $t=T_{f}$; afterwards the adjoint problem is integrated backwards in time. The initial condition for the adjoint problem, at $t=T_{f}$, is $\boldsymbol{u}^{\dagger}=\boldsymbol{u}$, whereas the direct problem is initiated, at $t=T_{0}$, with $\boldsymbol{u}=\boldsymbol{u}^{\dagger}$. The perturbations are normalised such that the energy is equal to one at $t=T_{0}$. The direct-adjoint iterative procedure is stopped when the energy $E\left(T_{f}\right)$ has converged to a value, with a precision below $10^{-8}$.

The equations are solved with a Chebyshev spectral collocation method in the inhomogeneous direction $(y)$. The Gauss-Lobatto-Chebyshev grid $\xi$ is expanded from the interval $[-1,1]$ to the physical domain $\left[0, y_{\infty}\right]$ through a linear mapping, 
(a)

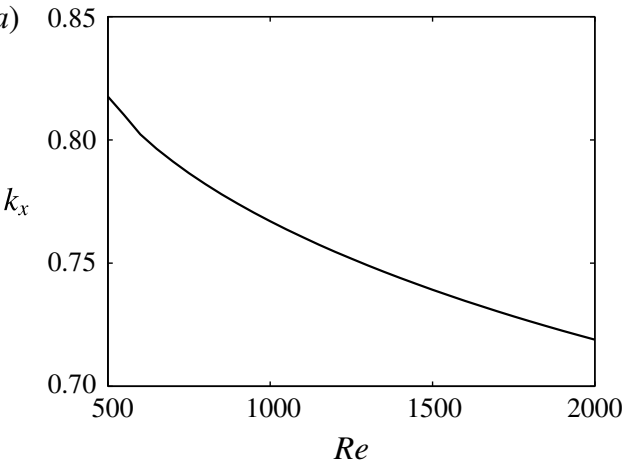

(b)

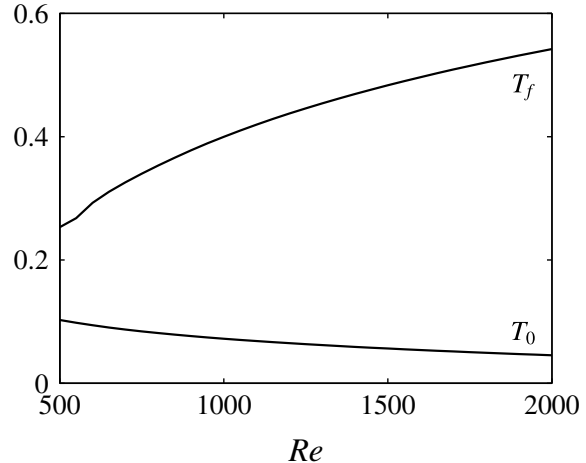

FIGURE 2. Optimal parameters for various Reynolds numbers: optimal streamwise wavenumber $(a)$ and temporal interval $(b)$. The optimal spanwise wavenumber is zero.

$y=y_{\infty}(1-\xi) / 2$. The divergence-free condition is achieved with a predictionprojection scheme. The pressure is approximated by polynomials of two units lower order than for the velocity, in order to compute a pressure unpolluted by spurious modes with only one collocation grid (Botella 1997). The time marching combines a fourth-order Adams-Bashforth scheme and a fourth-order backward differentiation scheme, with the viscous term treated implicitly (Ascher, Ruuth \& Wetton 1995). The numerical parameters are $y_{\infty}=10, N_{y}=100$ and $\Delta t=10^{-5}$.

\section{Results}

As preliminary results, the neutral stability results are obtained with $T_{0}=0, k_{z}=0$ and $T_{f}=4$. The critical Reynolds number is obtained when the final energy is close to the initial energy $E\left(T_{f}\right)=E\left(T_{0}\right)$, provided that $T_{f}$ is sufficiently large. The computed values are $R e_{c}=2511$ and $k_{x}=0.667$, in line with the values obtained by Blennerhassett \& Bassom (2002) and Thomas et al. (2010).

In the subcritical regime, the parametric study is realised by varying the five parameters: the streamwise $\left(k_{x}\right)$ and spanwise $\left(k_{z}\right)$ wavenumbers, the initial $\left(T_{0}\right)$ and final $\left(T_{f}\right)$ times, and the Reynolds number. For all cases, the optimal perturbation is two-dimensional; in other words, the spanwise velocity component and the spanwise wavenumber are both zero.

The optimal streamwise wavenumber is depicted in figure 2(a); the optimal value remains close to the critical value $\left(k_{x}=0.667\right)$. For low Reynolds number values, the optimal time to inject the perturbation, namely $T_{0}$, does not exactly correspond to the beginning of the deceleration phase $(t=0)$, see figure $2(b)$. Indeed, when the deceleration starts, the mean shear is primarily located close to the wall, where viscous damping is more effective; as a result, amplification is hampered. This results in a compromise for the optimal start of the Orr mechanism. For relatively large Reynolds numbers, the optimal part of the cycle, shown in figure $2(b)$, spreads throughout half a period: starting from the beginning of a deceleration phase up to the end of the next acceleration phase.

The maximum energy amplification increases exponentially with the Reynolds number (see figure 3). This scaling is characteristic of velocity profiles with reverse flow, for example the flow over a backward-facing step (Blackburn, Barkley \& Sherwin 2008) and the flow through an expanding pipe (Cantwell, Barkley \& 


\section{Biau}

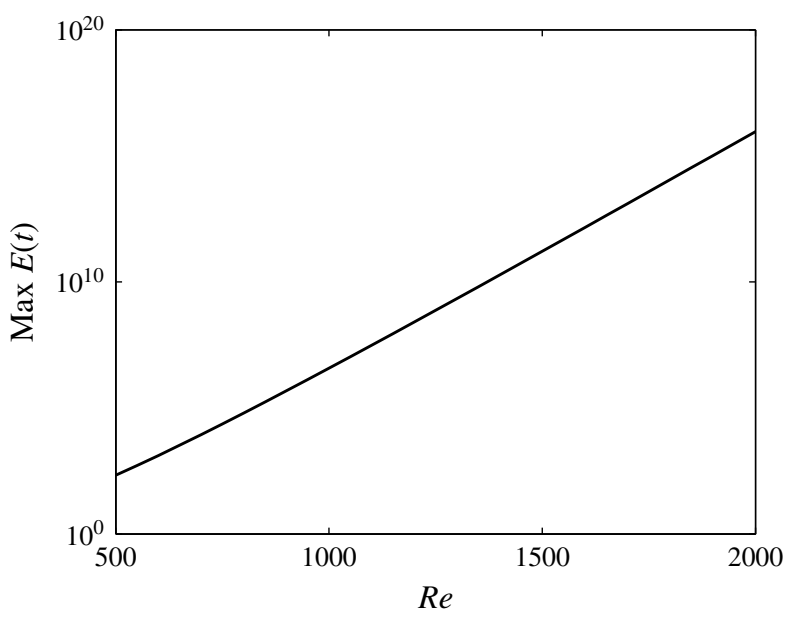

FIGURE 3. Maximum transient energy growth for various Reynolds numbers.

The perturbations are normalised such that $E\left(t=T_{0}\right)=1$.

Blackburn 2010). The transient growth of energy is fairly large, so that the modal path could be bypassed in supercritical regimes and replaced by this linear Orr mechanism, even for low disturbance levels.

The present results differ significantly from those obtained for steady shear flows (Butler \& Farrell 1992; Schmid \& Henningson 1999). In the latter case, streamwise vortices (with $k_{x}=0$ and $k_{z} \neq 0$ ) transform into streaks. The maximum disturbance energy, mostly carried by the streaks, scales as the square of the Reynolds number increases, which is much lower than the scale obtained for oscillatory flows. Moreover, for steady flows, the effect of acceleration is to damp growth (Corbett \& Bottaro 2000); in the present case the perturbations seem to be less sensitive to this damping effect.

The underlying mechanism for the transient amplification in oscillatory flows corresponds to an Orr mechanism, as can be seen in figure 4.

The mean shear is initially tilted against the direction of the initial disturbances. In this configuration, the structure borrows energy from the mean flow via the Reynolds stress production term (see (2.7)). The inclination of the spanwise vortices (figure 4) illustrates that the term $\overline{u v}$ is negative as long as the shear $\partial U / \partial y$ is positive. After half a period, the mean flow is tilted and aligned with the vorticity of the perturbation; as a consequence, the energy of the disturbances decreases. The transient growth mechanism is presented for the oscillating pressure gradient case, but the same mechanism is observed for the case of an oscillating wall.

In order to complete the qualitative results, values are presented in table 1, in particular for the $R e=1000$ case, which corresponds to the observed value for transition to turbulence.

The convergence of the present numerical method has been assessed by comparing the maximum energy growth computed with refined numerical parameters: $y_{\infty}=15$, $N_{y}=200$ and $\Delta t=10^{-6}$. For example, at $R e=1000$, the obtained value is $E\left(T_{f}\right)=3.7741 \times 10^{6}$. Comparison with the value in table 1 gives an estimate for the convergence to four significant digits.

Nonlinear simulations have been conducted at $R e=1000$ and 1200 , with the optimal parameters given in table 1 . In the following, the flow is driven by an oscillating 
(a)

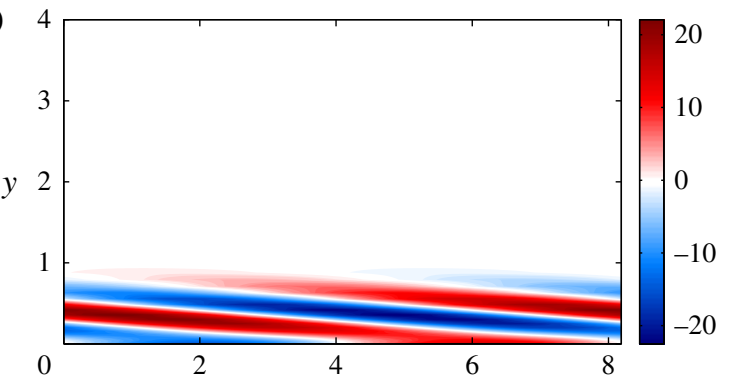

(c)

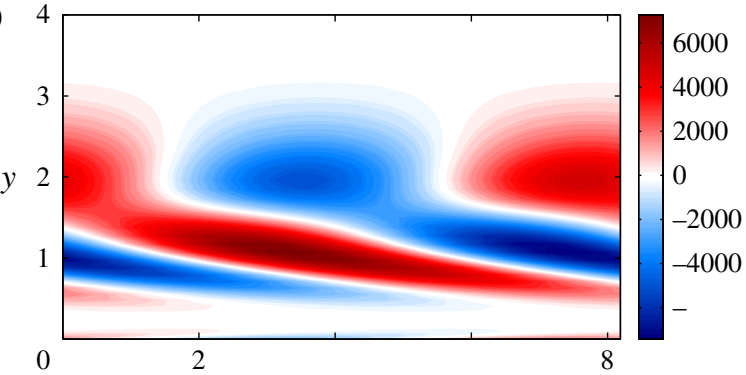

(b)

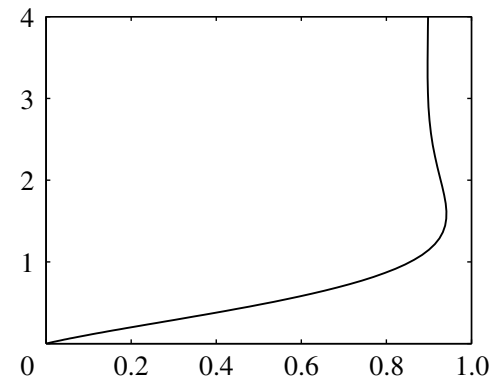

(d)

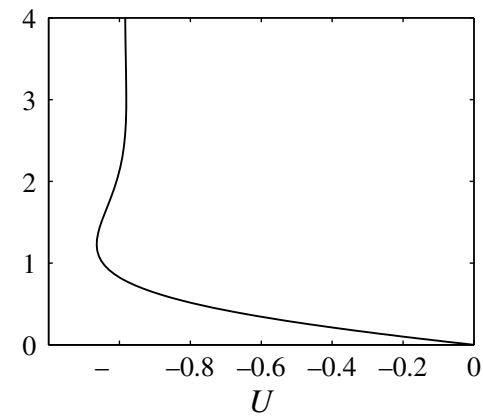

FIGURE 4. Contours of the spanwise vorticity of the perturbations and the corresponding base flow profile for $t=T_{0}(a, b)$ and $t=T_{f}(c, d)$, at $R e=1000$.

$\begin{array}{rccccc}R e & k_{x} & k_{z} & T_{0} & T_{f} & E\left(T_{f}\right) \\ 800 & 0.7821 & 0 & 0.0815 & 0.3532 & 6.2834 \times 10^{4} \\ 1000 & 0.7670 & 0 & 0.0723 & 0.3999 & 3.7743 \times 10^{6} \\ 1200 & 0.7546 & 0 & 0.0650 & 0.4376 & 2.5692 \times 10^{8}\end{array}$

TABLE 1. Optimal parameters for three Reynolds number values.

pressure gradient, but the results are qualitatively similar to those obtained with an oscillating wall (not shown). The streamwise and spanwise lengths of the domain are $2 \pi / k_{x}$, with $k_{x}$ the optimal streamwise wavenumber. The numerical parameters are $y_{\infty}=10, N_{y}=100, \Delta t=10^{-5}$ and $N_{x}=N_{z}=128$ Fourier modes in the homogeneous directions. These numerical parameters are consistent with those used by Ozdemir et al. (2014). The initial states consist of the unsteady base flow profile plus the optimal perturbation, normalised with the prescribed energy $E_{0}=10^{-7}$ and $E_{0}=10^{-9}$ for $R e=1000$ and 1200 respectively. A white Gaussian divergence-free noise is superimposed in order to break the spanwise invariance, with a prescribed energy $E_{1}=10^{-3} E_{0}$. The two simulations similarly lead to turbulent flows; the case $R e=1000$ is illustrated in figure 5 .

In figure 5, the evolution energy of the perturbations obtained from the threedimensional simulation is compared with the two-dimensional case and the linear evolution. The nonlinear evolution follows the linear curve over four decades, at which point the base flow is slightly distorted by quadratic interaction. As a consequence, the amplification saturates and the maximal energy gain does not reach the linear value. While the two-dimensional simulation shows a relaminarisation, 


\section{Biau}

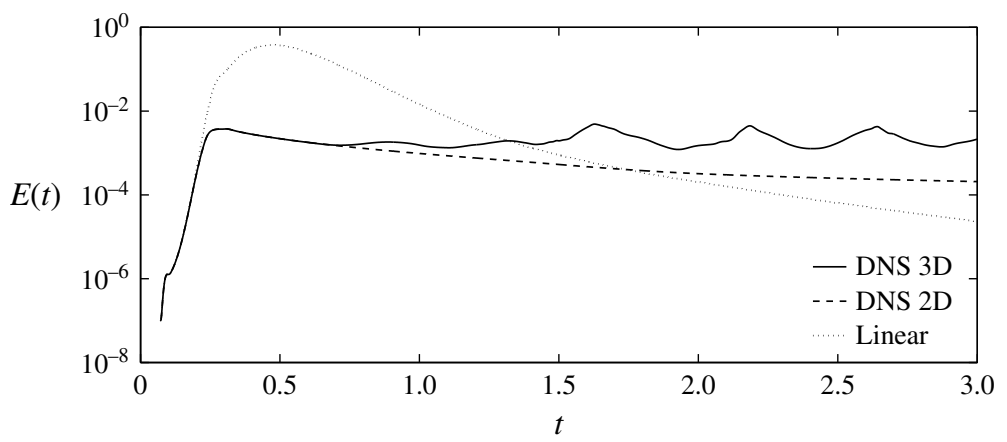

FIgURE 5. The temporal evolution of the kinetic energy of the perturbations, at $R e=1000$, computed in three different frameworks, namely the three-dimensional nonlinear simulation (DNS 3D), the two-dimensional nonlinear simulation (DNS 2D) and the linear evolution.

with a slow decay of the mean flow defect, the three-dimensional case shows that the two-dimensional wave destabilises into three-dimensional disturbances, with an evolution towards turbulence. As for steady flows, three-dimensional fluctuations are necessary to observe a self-sustaining wall turbulence in subcritical regimes. Indeed, as a common feature of the near-wall turbulence, the development of streamwise low-speed streaks is observed at the end of the transition to turbulence (Jimènez \& Moin 1991; Hamilton, Kim \& Waleffe 1995). The amplification is fast; the whole transition can be realised within one deceleration phase. Following the transient, the flow is close to the laminar state in the accelerated phase, but turbulent bursts are triggered during the decelerated phase of the cycle. This transition towards turbulence is similar to the simulations initialised with non-optimal perturbations by Ozdemir et al. (2014).

\section{Conclusion}

The subcritical instability of oscillatory Stokes flows was investigated with a linear optimal perturbation analysis. The parametric study indicates that the disturbances are able to efficiently extract energy from the mean shear by transporting momentum down the mean velocity gradient. The results show a strong Orr mechanism, with amplifications increasing exponentially with the Reynolds number. The optimal disturbances take the form of two-dimensional spanwise vortices, with streamwise wavenumber close to the value $k_{x}=0.7$.

The optimisation strategy completes the modal (asymptotic) analysis by Blennerhassett \& Bassom (2002), in particular for subcritical Reynolds numbers. As the Orr mechanism is non-modal by nature, this process can only be observed by considering an initial value problem. Nonetheless, an optimisation strategy for computing the initial condition raises the issue of the frequency of occurrence of such an event. While the unstable mode eventually emerges in supercritical conditions, the transient amplification results in a subtle combination of eigenmodes, determined by the receptivity process.

The present results, applied to classical oscillatory boundary layer flows, can be extended to more complex flows by using a linear combination of the Stokes solutions. For example, Spalart (1989) used Stokes solutions for the velocity components in the streamwise and spanwise directions in order to create simple turbulent boundary layer 


\section{Transient growth in oscillatory flows}

flows with three-dimensional statistics. A possible extension of the research presented in the present paper would be the stability analysis of the superposition of two Stokes solutions, with an irrational frequency ratio.

\section{References}

AKhaVAn, R., KAMm, R. D. \& Shapiro, A. H. 1991 An investigation of transition to turbulence in bounded oscillatory Stokes flows. Part 2. Numerical simulations. J. Fluid Mech. 225, 423-444.

Andersson, P., Berggren, M. \& Henningson, D. S. 1999 Optimal disturbances and bypass transition in boundary layers. Phys. Fluids 11, 134-150.

Ascher, U. M., RuUth, S. J. \& Wetton, B. T. R. 1995 Implicit-explicit methods for timedependent partial differential equations. SIAM J. Numer. Anal. 32, 797-823.

Blackburn, H. M., Barkley, D. \& Sherwin, S. J. 2008 Convective instability and transient growth in flow over a backward-facing step. J. Fluid Mech. 603, 271-304.

Blennerhassett, P. J. \& Bassom, A. P. 2002 The linear stability of flat Stokes layers. J. Fluid. Mech. 464, 393-410.

Blennerhassett, P. J. \& Bassom, A. P. 2008 On the linear stability of Stokes layers. Phil. Trans. R. Soc. Lond. A 366, 2685-2697.

BlondeauX, P. \& VitToRI, G. 1994 Wall imperfections as a triggering mechanism for Stokes-layer transition. J. Fluid Mech. 264, 107-135.

Botella, O. 1997 On the solution of the Navier-Stokes equations using Chebyshev projection schemes with third-order accuracy in time. Comput. Fluids 26, 107-116.

Butler, K. M. \& FARrell, B. F. 1992 Three-dimensional optimal perturbations in viscous shear flow. Phys. Fluids A 4, 1637-1650.

Cantwell, C. D., Barkley, D. \& Blackburn, H. M. 2010 Transient growth analysis of flow through a sudden expansion in a circular pipe. Phys. Fluids 22, 034101.

Conrad, P. W. \& CRiminale, W. O. 1965 The stability of time-dependent laminar flow: parallel flows. Z. Angew. Math. Phys. 16, 233-254.

Corbett, P. \& BotTARo, A. 2000 Optimal perturbations for boundary layers subject to stream-wise pressure gradient. Phys. Fluids 12, 120-130.

FARrell, B. F. 1988 Optimal excitation of perturbations in viscous shear flow. Phys. Fluids 31, 2093-2102.

Hall, P. 1978 The linear stability of flat Stokes layers. Proc. R. Soc. Lond. A 359, 151-166.

Hamilton, J. M., Kim, J. \& WALEFFE, F. 1995 Regeneration mechanisms of near-wall turbulence structures. J. Fluid Mech. 287, 317-348.

JimèneZ, J. \& MoIn, P. 1991 The minimal flow unit in near-wall turbulence. J. Fluid Mech. 225, 221-240.

LUCHINI, P. 2000 Reynolds number independent instability of the boundary layer over a flat surface: optimal perturbations. J. Fluid Mech. 404, 289-309.

Luchini, P. \& Bottaro, A. 2014 Adjoint equations in stability analysis. Annu. Rev. Fluid Mech. 46, 493-517.

ORR, W. 1907 The stability or instability of the steady motions of a perfect liquid and of a viscous liquid. Part II: a viscous liquid. Proc. R. Irish Acad. A 27, 1907-1909.

Ozdemir, C., Hsu, T.-J. \& BALAChandar, S. 2014 Direct numerical simulations of transition and turbulence in smooth-walled Stokes boundary layer. Phys. Fluids 26, 045108.

SCHLichting, H. 1979 Boundary-layer Theory. McGraw-Hill.

SChMid, P. J. 2007 Nonmodal stability theory. Annu. Rev. Fluid Mech. 39, 129-162.

SChmid, P. J. \& Henningson, D. S. 1999 Stability and Transition of Shear Flows. Springer.

Spalart, P. R. 1989 Theoretical and numerical study of a three-dimensional turbulent boundary layer. J. Fluid Mech. 205, 319-340.

Thomas, C., Blennerhassett, P. J., Bassom, A. P. \& Davies, C. 2015 The linear stability of a Stokes layer subjected to high-frequency perturbations. J. Fluid Mech. 764, 193-218.

Thomas, C. T., Bassom, A. P., Blennerhassett, P. J. \& Davies, C. 2010 Direct numerical simulations of small disturbances in the classical Stokes layer. J. Engng Maths 68, 327-338. 


\section{Biau}

Trefethen, L. N., Trefethen, A. E., Reddy, S. C. \& Driscoll, T. A. 1993 Hydrodynamic stability without eigenvalues. Science 261, 578-584.

VitToRi, G. \& VERzicco, R. 1998 Direct simulation of transition in an oscillatory boundary layer. J. Fluid Mech. 371, 207-232.

Von KerczeK, C. \& DAVIS, S. H. 1974 Linear stability theory of oscillatory Stokes layers. J. Fluid Mech. 62 (4), 753-773.

Zhao, M., Ghidaoui, M. S. \& Kolyshkin, A. A. 2007 Perturbation dynamics in unsteady pipe flows. J. Fluid Mech. 570, 129-154. 